\title{
Elastic anysotropy of dentin and enamel
}

\author{
S. A. Muslov ${ }^{1, \dagger}$, D. S. Lisovenko ${ }^{2}$ \\ †muslov@mail.ru \\ ${ }^{1}$ Evdokimov Moscow State Medical Stomatological University (MSMSU), 20 - 1 Delegatskaya str., Moscow, 127473, Russia \\ ${ }^{2}$ Ishlinsky Institute for Problems in Mechanics RAS, 101 - 1 Vernadskogo ave., Moscow, 119526, Russia
}

\begin{abstract}
The anisotropy of elastic properties of hard tooth tissues - dentine and enamel are studied. Dentin and enamel are considered as anisotropic media with a hexagonal symmetry, elastic properties of which are described by an appropriate matrix of elastic moduli and compliance factors. The Poisson's ratio and parameters of elastic anisotropy are calculated. Extremum values of Poisson's ratio for dentin and enamel and Poisson's ratios for particular orientations are found. The calculations show that these parameters exceed the boundary values characteristic of isotropic materials including the common composite materials used in dentistry for restoration. It is assumed that this can result in the formation of overstress domains on the filling material-dentin and filling material-enamel interfaces, facilitation the growth of microcracks and weaken the material adhesion to dentin and enamel thus destroying the results of restoration by composite materials. The elastic anisotropy of hexagonal crystals was determined by means of several parameters assuming, basing on literature data, that there is no such an anisotropy index of hexagonal crystals deformation that could be accepted as the main one. Orientation dependencies of the elastic moduli are constructed. For hard tooth tissues and skeleton bones, which are composites, i.e. microinhomogeneous materials, the possibility of predicting «technical» elastic characteristics on the basis of the measurement and calculation of the elastic parameters of their individual components is assessed. The data obtained are expected to help better understanding of correlation between the structure and elastic properties of tissues and to optimize solution of problems in dentition biomechanics taking into account the symmetry of the mechanical properties.
\end{abstract}

Keywords: elastic properties, anisotropy, Poisson's ratio, dentin, enamel, hydroxyapatite.

УДК: 539.3

\section{Упругая анизотропия дентина и эмали}

\author{
Муслов С. А. ${ }^{1, \dagger}$, Лисовенко Д. С. ${ }^{2}$ \\ ${ }^{1}$ Московский государственный медико-стоматологический университет (МГМСУ) им. А.И. Евдокимова, \\ ул. Делегатская 20 - 1, Москва, 127473, Россия \\ ${ }^{2}$ Институт проблем механики им. А. Ю. Ишлинского РАН, пр-т Вернадского 101 - 1, Москва, 119526, Россия
}

Исследована анизотропия упругих свойств твердых тканей зуба - дентина и эмали. Дентин и эмаль рассмотрены как анизотропные среды гексагональной симметрии, упругие свойства которых описываются соответствующей матрицей упругих постоянных или коэффициентов податливости. Рассчитаны коэффициенты Пуассона и параметры упругой анизотропии. Получены экстремальные значения коэффициента Пуассона для дентина и эмали, а также значения коэффициентов Пуассона в частных ориентациях. По результатам расчетов отмечено, что данные параметры превышают характерные граничные значения, присущие изотропным материалам, в том числе известным композиционным, используемым в стоматологии для реставрации. Предположено, что это может приводить к образованию доменов перенапряжений на границе пломбировочный материал-дентин и пломбировочный материалэмаль, сопутствовать росту микротрещин, ослаблять сцепление материала с дентином и эмалью, разрушать результаты реставрации композиционными материалами. Упругую анизотропию гексагональных кристаллов определяли через несколько параметров, поскольку на основании литературных данных считали, что нет такого показателя анизотропии деформации гексагональных кристаллов, который можно бы было считать основным. Также были построены ориентационные зависимости упругих модулей материалов. Оценена возможность предсказания “технических" упругих характеристик микронеоднородных материалов - композитов, какими являются твердые ткани зуба и кости скелета, на основе измерения и расчетов упругих параметров отдельных их компонентов. Представляется, что полученные данные будут способствовать лучшему пониманию вопроса о взаимосвязи архитектоники зуба и его механических свойств, проблем прочности биокомпозитов, а также оптимизации решения задач биомеханики зубочелюстной системы с учетом упругой симметрии составляющих её твердых тканей.

Ключевые слова: упругие свойства, упругие постоянные, анизотропия, коэффициент Пуассона, дентин, эмаль, гидроксиапатит. 


\section{1. Введение}

Кристаллы гидроксиапатита $\mathrm{Ca}_{10}\left(\mathrm{PO}_{4}\right)_{6}(\mathrm{OH})_{2}$, минеральной основы костной ткани, эмали и дентина, имеют гексагональную кристаллическую структуру. Упругие свойства твердых тканей зуба и гидроксиапатита, как минеральной их составляющей, достаточно часто вызывали интерес у исследователей [1-4]. Тем не менее, остается актуальным вопрос о структурной организации твердых тканей зуба, представляющий собой минерально-органический комплекс и гетерофазную систему и вопрос о её влиянии на структурно-чувствительные упругие характеристики. Также представляет интерес возможность предсказания упругих свойств микронеоднородных материалов - поликристаллов и композитов (какими являются твердые ткани зуба и кости скелета) на основе измерения и расчетов упругих характеристик отдельных их компонентов.

Модуль Юнга и коэффициент Пуассона являются постоянными величинами для изотропного материала и полностью характеризуют его упругие свойства. Для анизотропных материалов модуль Юнга и коэффициент Пуассона, которые выражаются через коэффициенты податливости $s_{i j}$ или жесткости $c_{i j}$, зависят от ориентации кристаллической решетки. В случае гексагональных систем упругие свойства описываются пятью независимыми коэффициентами жесткости или податливости: $c_{11}, c_{12}, c_{13}, c_{33}, c_{44}$ или $s_{11}, s_{12}, s_{13}, s_{33}, s_{44}$. Для гексагональных кристаллов также существует связь $c_{66}=0.5\left(c_{11}-c_{12}\right)$ или $s_{66}=2\left(s_{11}-s_{12}\right)[5,6]$.

\section{2. Коэффициент Пуассона}

Известно, что коэффициент Пуассона характеризует отношение поперечной деформации к продольной и является одной из упругих констант материала. Коэффициент Пуассона для изотропных материалов меняется в пределе от -1 до 0.5 [7]. Для обычных изотропных материалов его значения лежат в интервале от 0 до 0.5 . Известны также материалы с отрицательным коэффициентом Пуассона [8-13]. Для дентина по литературным данным коэффициент Пуассона принимает значения в интервале $0.30-0.32$ [14]. Субмикроскопическая структура дентина как композитного материала представлена кристаллами гидроксиапатита, которые расположены между коллагеновыми волокнами и по классу симметрии относятся к гексагональной сингонии. Минеральная основа эмали зубов представлена гексагональными кристаллами гидрокси-, карбонат-, хлор-, фторапатитов. Эмалевые призмы являются основным структурным образованием. Органическое вещество эмали имеет вид тончайших фибриллярных структур, которые по существующему мнению, определяют ориентацию кристаллов эмалевой призмы. Поэтому есть все основания полагать рассматривать дентин и эмаль как анизотропные среды, упругие свойства которых описываются матрицей упругих постоянных $c_{i j}$ или коэффициентов податливости $s_{i j}$. По литературным данным значения упругих постоянных эмали и дентина достаточно хорошо известны [15]. Поэтому зная их, можно вычислить коэффициенты податливости и «технические» упругие характеристики (модуль Юнга, коэффициент Пуассона и модуль сдвига) дентина по формулам для гексагональной сингонии с использованием связи между коэффициентами податливости и жесткости [5]:

$$
\begin{gathered}
c_{11}+c_{12}=\frac{s_{33}}{s}, \quad c_{11}-c_{12}=\frac{1}{s_{11}-s_{12}}, \quad c_{13}=-\frac{s_{13}}{s}, \\
c_{33}=\frac{s_{11}+s_{12}}{s}, \quad c_{44}=\frac{1}{s_{44}}, \quad c_{66}=\frac{1}{s_{66}}, \\
s=s_{33}\left(s_{11}+s_{12}\right)-2 s_{13}^{2} .
\end{gathered}
$$

Значения коэффициентов податливостей дентина и эмали представлены в Табл. 1.

Модуль Юнга $E$, коэффициент Пуассона $v$ и модуль сдвига $G$ для гексагональных кристаллов можно записать в виде [16,17]

$$
\begin{gathered}
\frac{1}{s_{11} E}=1+\left(\Pi_{1}-\Pi_{01} \sin ^{2} \theta\right) \cos ^{2} \theta \\
-\frac{v}{s_{13} E}=1+\left(\Pi_{2} \cos ^{2} \psi+\Pi_{02} \cos ^{2} \theta \sin ^{2} \psi\right) \sin ^{2} \theta \\
\frac{1}{s_{44} G}=1+\left(\Pi_{3} \cos ^{2} \psi+4 \Pi_{03} \cos ^{2} \theta \sin ^{2} \psi\right) \sin ^{2} \theta \\
\Pi_{01} \equiv \frac{\delta}{s_{11}}, \quad \Pi_{02} \equiv \frac{\delta}{s_{13}}, \quad \Pi_{03} \equiv \frac{\delta}{s_{44}} \\
\Pi_{1} \equiv \frac{s_{33}-s_{11}}{s_{11}}, \quad \Pi_{2} \equiv \frac{s_{12}-s_{13}}{s_{13}}, \quad \Pi_{3} \equiv \frac{2 s_{11}-2 s_{12}-s_{44}}{s_{44}} \\
\delta \equiv s_{11}+s_{33}-2 s_{13}-s_{44} .
\end{gathered}
$$

Результаты вычисления коэффициентов Пуассона дентина и эмали как кристаллических гексагональных структур представлены на Рис. 1 и в Табл. 2.

Табл. 1. Коэффициенты податливости (в ТПа $\left.{ }^{-1}\right)$ дентина и эмали. Table 1. The compliance coefficients (in $\mathrm{TPa}^{-1}$ ) of dentin and enamel.

\begin{tabular}{|c|c|c|c|c|c|}
\hline $\begin{array}{c}\text { Материал } \\
\text { Мaterial }\end{array}$ & $s_{11}$ & $s_{12}$ & $s_{13}$ & $s_{33}$ & $s_{44}$ \\
\hline $\begin{array}{c}\text { Дентин } \\
\text { Dentin }\end{array}$ & 34.6 & -14.5 & -4.5 & 27.6 & 175.4 \\
\hline $\begin{array}{c}\text { Эмаль } \\
\text { Еnamel }\end{array}$ & 10.4 & -3.4 & -1.7 & 8.8 & 43.9 \\
\hline
\end{tabular}

Табл. 2. Экстремальные значения коэффициента Пуассона $\left(v_{\min }, v_{\max }\right)$ для дентина и эмали, а такжезначения коэффициентов Пуассона в частных ориентациях (последние четыре цифры в квадратных скобках указывают направление растяжения, а первых четыре - направление поперечной деформации).

Table 2. Extremal values of Poisson's ratio $\left(v_{\min }, v_{\max }\right)$ and Poisson's ratios in particular orientations for dentin and enamel (the last four digits in square brackets indicate the direction of stretching, and the first four digits indicate the direction of the transverse deformation).

\begin{tabular}{|c|c|c|c|c|c|}
\hline $\begin{array}{c}\text { Материал } \\
\text { Мaterial }\end{array}$ & $v_{\max }$ & $v_{\min }$ & $v_{[2 \overline{1} \overline{1} 0],[0001]}$ & $v_{[01 \overline{1} 0],[2 \overline{1} \overline{1} 0]}$ & $v_{[0001],[2 \overline{1} \overline{1} 0]}$ \\
\hline $\begin{array}{c}\text { Дентин } \\
\text { Dentin }\end{array}$ & 0.54 & 0.13 & 0.16 & 0.13 & 0.42 \\
\hline $\begin{array}{c}\text { Эмаль } \\
\text { Еnamel }\end{array}$ & 0.47 & 0.16 & 0.19 & 0.16 & 0.33 \\
\hline
\end{tabular}




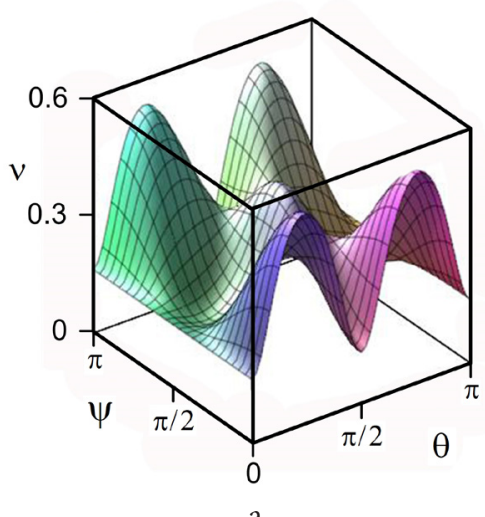

a

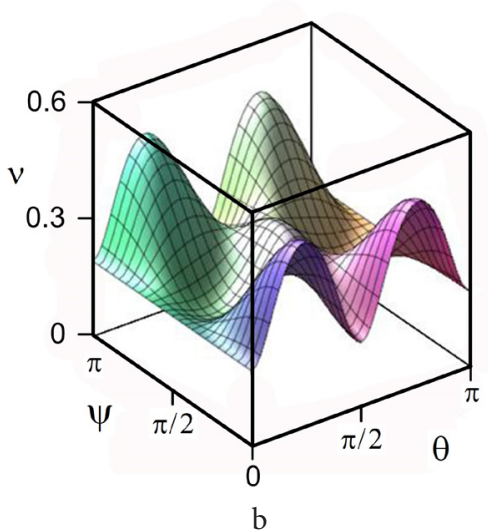

Рис. 1. Изменчивость коэффициентов Пуассона для дентина (а) и эмали (b).

Fig. 1. Variability of Poisson's ratios for dentin (a) and enamel (b).

Поверхности коэффициента Пуассона для дентина и эмали на Рис. 1 подобны. Из них отчетливо следует выраженное анизотропное поведение коэффициентов Пуассона. Его значения в зависимости от направления (углов Эйлера $\theta$ и $\psi$ ) изменяются в широких пределах (до четырех с лишним раз). Направления растяжения $\mathbf{n}$ и поперечной деформации $\mathbf{m}$ можно определить с помощью следующих формул

$$
\begin{gathered}
\mathbf{n}=\left(\begin{array}{c}
\sin \varphi \sin \theta \\
-\cos \varphi \sin \theta \\
\cos \theta
\end{array}\right) \\
\mathbf{m}=\left(\begin{array}{c}
\cos \varphi \cos \psi-\sin \varphi \cos \theta \sin \psi \\
\sin \varphi \cos \psi+\cos \varphi \cos \theta \sin \psi \\
\sin \theta \sin \psi
\end{array}\right) .
\end{gathered}
$$

Отметим также аномально высокую величину максимального значения коэффициента Пуассона (0.54) вдоль ряда направлений, что оказывается выше значения верхнего предела для коэффициента Пуассона изотропных материалов. Это означает, что при сжатии локальных областей дентина и эмали вдоль этих направлений их объем будет увеличиваться, а при растяжении - наоборот, уменьшаться. Упругое поведение под нагрузкой пломбировочного материала принципиально другое (коэффициент Пуассона существенно меньше 0.5), поэтому при сжатии его объем уменьшается, а при растяжении увеличивается. Это может привести к образованию областей перенапряжений на границе пломбировочный материал-дентин и пломбировочный материал-эмаль, сопутствующих росту микротрещин, ослаблению сцепления материала с дентином и эмалью, нарушению краевого прилегания пломбы, деградации фиксации и часто встречаемой ситуации разрушения прямой, а порой и непрямой реставрации, особенно композиционными материалами.

\section{3. Параметры анизотропии}

В настоящее время пристальное внимание привлекает изучение процессов микронеоднородного деформирования, так как эти процессы определяют закономерности процессов зарождения пластических деформаций и возникновения микротрещин при различных видах нагружения. Большинство конструкционных материалов являются поликристаллическими телами, то есть состоят из большого числа кристаллитов, обладающих анизотропией упругих, пластических, прочностных и других свойств. Взаимодействие анизотропных кристаллитов приводит к возникновению в микрообъёмах сложного напряжённо-деформированного состояния [18]. Можно отметить, что чем больше различия экстремальных значений модулей упругости, тем больше уровень концентрации напряжений. Вследствие этого изучение показателей анизотропии неоднородных сред приобретает весьма большое значение при изучении проблем прочности.

Только одновременно учитывая их величины, можно проводить аналогии и прогнозировать изменение параметров, характеризующих степень концентрации микронапряжений, ответственных за возникновение пластических деформаций и микротрещин, в зависимости от степени анизотропии.

В отличие от кубических кристаллов, анизотропия упругих свойств которых может быть описана одним показателем $2 c_{44} /\left(c_{11}-c_{12}\right)$, упругую анизотропию гексагональных кристаллов определяют несколько параметров, при этом нет такого показателя анизотропии деформации гексагональных кристаллов, который можно бы было считать основным.

Если проанализировать выражения для компонент тензоров упругих постоянных или коэффициентов податливости, содержащих направляющие косинусы с множителями, то будет очевидно, что степень анизотропии будет определяться отличием последних от нуля. То есть параметры анизотропии можно характеризовать отношениями

$$
\begin{gathered}
U=\frac{c_{11}+c_{33}+2 c_{13}+4 c_{44}}{4 c_{44}}, \quad R=\frac{-c_{11}+2 c_{44}+c_{13}}{2 c_{44}}, \\
Q=\frac{-c_{12}+c_{13}}{2 c_{44}} .
\end{gathered}
$$

Таким образом, для гексагональной кристаллической решетки число независимых параметров анизотропии можно считать равным трем [19].

По мнению [20] существует три независимых упругих сдвига в гексагональных кристаллах; так что анизотропию к упругому сдвигу можно представить тремя показателями (тремя отношениями жесткостей к сдвигам): 


$$
A_{1}=\frac{c_{11}+c_{12}+2 c_{33}-4 c_{13}}{6 c_{44}}, \quad A_{2}=\frac{2 c_{44}}{c_{11}-c_{12}}, \quad A_{3}=A_{1} A_{2} .
$$

Еще один полезный показатель анизотропии упругой деформации при растяжении может быть получен из отношения модулей Юнга в направлениях, перпендикулярном и параллельном оси шестого порядка:

$$
A_{4}=\frac{S_{33}}{S_{11}} .
$$

При этом по мнению авторов наиболее наглядный показатель анизотропии деформации при всестороннем сжатии:

$$
A_{5}=\frac{2 s_{13}+s_{33}}{s_{11}+s_{12}+s_{13}} .
$$

На основании предложенных в литературе подходов для гексагональных кристаллов были проанализированы численные значения всех описанных параметров анизотропии для дентина и эмали. Данные анализа представлены в Табл. 3. Помимо дентина и эмали в расчеты были включены данные по упругим свойствам костной тканей (В) и кристаллов гидроксиапатита (Нар) [15].

В результате можно сделать следующие выводы. Итоговая наибольшая упругая анизотропия наблюдалась по параметрам $U$ и $R$, наименьшая - по $Q$ (см. Табл. 3). Наибольшая анизотропия упругих свойств имеет место по параметрам $U, R$ и $Q$ у дентина и эмали, меньшая у кости и наименьшая - у гидроксиапатита. Среди по- казателей $A_{1}-A_{5}$ установлена наибольшая анизотропия по параметрам $A_{1}$ и $A_{3}$, наименьшая по $A_{2}$ и $A_{4}$. Наибольшая анизотропия, судя по параметрам $A_{1}-A_{5}$, обнаружена снова у дентина и эмали, меньшая - у кости и наименьшая - у гидроксиапатита.

Независимо от того какие группы независимых параметров были использованы наибольшей упругой анизотропией обладают дентин, эмаль, костная ткань и наименьшей - кристаллы гидроксиапатита (Нар). Таким образом, установлено, что коллагеновый и фибриллярный матрикс, входящий в состав исследованных биокомпозитов как органическая компонента, увеличивает анизотропию упругих свойств кальций-фосфатных материалов, что, в общем, является достаточно неожиданным результатом и требует дальнейшего исследования. Возможно, органическая составляющая существенно упорядочена, что увеличивает анизотропию свойств биокомпозитного комплекса в состав которого она входит.

\section{4. Анизотропия упругих модулей}

Также были построены ориентационные зависимости

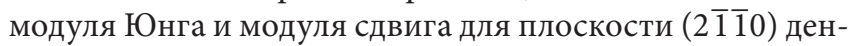
тина, эмали, костной ткани и гидроксиапатита (Рис. 2). Ориентационные зависимости служат наглядным графическим представлением анизотропии физических свойств.

Табл. 3. Значения параметров анизотропии для дентина, эмали, костной ткани и гидроксиапатита (Нар).

Table. 3. The values of the anisotropy parameters for dentin, enamel, bone tissue and hydroxyapatite (Hap).

\begin{tabular}{|c|c|c|c|c|c|c|c|c|}
\hline Материал / Material & $U$ & $R$ & $Q$ & $A_{1}$ & $A_{2}$ & $A_{3}$ & $A_{4}$ & $A_{5}$ \\
\hline Кость (В) / Bone & 0.67 & -0.59 & -0.28 & 1.79 & 0.76 & 1.36 & 0.83 & 1.07 \\
\hline Нар & 0.26 & -0.04 & 0.16 & 1.28 & 0.84 & 1.07 & 0.83 & 0.60 \\
\hline Эмаль (Е) / Enamel & 0.97 & -0.86 & -0.27 & 2.10 & 0.63 & 1.32 & 0.85 & 1.03 \\
\hline Дентин (D) / Dentin & 1.57 & -1.48 & -0.69 & 2.83 & 0.56 & 1.58 & 0.80 & 1.19 \\
\hline
\end{tabular}
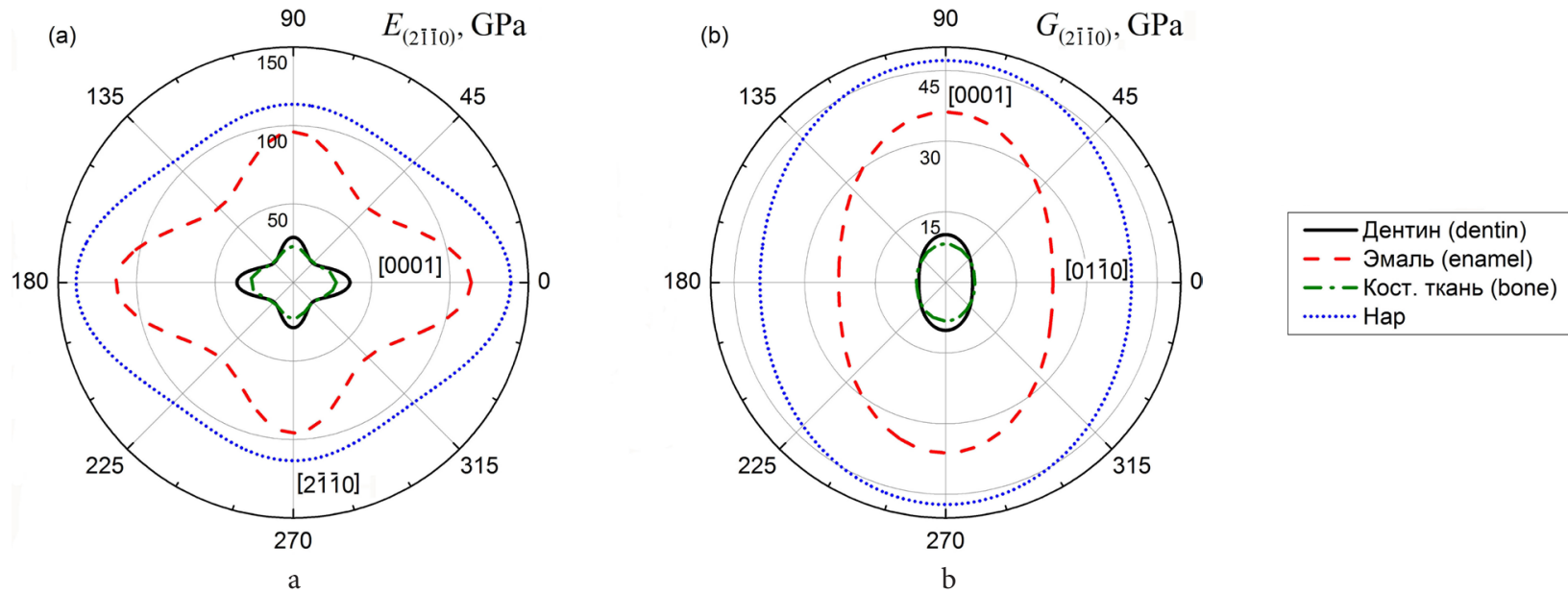

Рис. 2. Ориентационные зависимости модуля Юнга $E$ (a) и модуля сдвига $G(\mathrm{~b})$ дентина, эмали, костной ткани и гидроксиапатита для плоскости $(2 \overline{1} \overline{1} 0)$.

Fig. 2. Orientational dependences of Young's modulus $E$ (a) and the shear modulus $G$ (b) of dentin, enamel, bone tissue and hydroxyapatite for the plane $(2 \overline{1} \overline{1} 0)$. 
Как видно из рисунков вид сечений для модулей Юнга идентичен для всех исследованных материалов, хотя уровень упругих свойств гидроксиапатита и эмали заметно выше, чем у кости и дентина (см. Табл. 4). Что касается модуля сдвига, то кристаллы гидроксиапатита демонстрируют упругую анизотропию, обратную, чем костная ткань, эмаль и дентин.

\section{5. Предсказание упругих свойств дентина и эмали как микронеоднородных материалов}

С помощью хорошо известных методов усреднения удается определить средние значения упругих характеристик для поликристаллов со случайной ориентацией зерен. Согласно усреднению по Фойгту модуль всестороннего сжатия и модуль сдвига для гексагональных кристаллов равны соответственно

$$
\begin{gathered}
\langle K\rangle=\frac{2 c_{11}+c_{33}+2\left(c_{12}+2 c_{13}\right)}{9}, \\
\langle G\rangle=\frac{7 c_{11}+2 c_{33}-5 c_{12}-4 c_{13}+12 c_{44}}{30} .
\end{gathered}
$$

При подходе, предложенном Ройсом, производится усреднение тензора податливости, в результате которого для гексагональной решетки получаем

$$
\begin{gathered}
\left\langle\frac{1}{K}\right\rangle=2 s_{11}+s_{33}+2\left(s_{12}+2 s_{13}\right), \\
\left\langle\frac{1}{G}\right\rangle=\frac{2\left(7 s_{11}+2 s_{33}+3 s_{44}-5 s_{12}-42 s_{13}\right)}{15} .
\end{gathered}
$$

Обозначая модули упругости, полученные усреднением тензора упругости, через $K_{\mathrm{v}}$ и $G_{\mathrm{v}}$ (по Фойгту), а усреднением тензора податливости - через $K_{\mathrm{R}}$ и $G_{\mathrm{R}}$ (по Ройсу), можно записать следующие неравенства [18]

$$
K_{\mathrm{R}} \leq K_{э \phi \phi} \leq K_{\mathrm{V}}, \quad G_{\mathrm{R}} \leq G_{\ni ф \phi} \leq G_{\mathrm{V}}
$$

где через $K_{\text {эфф }}$ и $G_{\text {эфф }}$ обозначены эффективные модули упругости, связывающие усредненные напряжения и деформации. Именно эти модули измеряются экспериментально на поликристаллических образцах. Во многих случаях хорошее согласие с экспериментальными данными дает предложенное Хиллом среднее арифметическое значение, найденное усреднением по Фойгту и Ройсу, а именно

$$
K_{\mathrm{H}}=\frac{1}{2}\left(K_{\mathrm{V}}+K_{\mathrm{R}}\right), \quad G_{\mathrm{H}}=\frac{1}{2}\left(G_{\mathrm{V}}+G_{\mathrm{R}}\right) .
$$

Помня о том, что в изотропном приближении два модуля независимы, можно записать выражения для $E$ и $v$ как функции $K$ и $G$ :

$$
\langle E\rangle=\frac{9 K G}{3 K+G} \text { и }\langle v\rangle=\frac{3 K-2 G}{6 K+2 G} .
$$

В Табл. 5 приведены значения усредненных упругих модулей, рассчитанных по значениям упругих постоянных с помощью хорошо известных методов усреднения,

\begin{tabular}{|c|c|c|c|c|c|}
\hline \multicolumn{6}{|c|}{ Модуль Юнга / Young`s modulus } \\
\hline Материал / Material & $E_{\text {max }}, \mathrm{GPa}$ & $E_{\text {min }}, \mathrm{GPa}$ & $E_{\max } / E_{\min }$ & $E_{[01 \overline{1} 0]}, \mathrm{GPa}$ & $E_{[0001]}, \mathrm{GPa}$ \\
\hline Кость (B) / Bone & 27.4 & 17.8 & 1.54 & 22.8 & 27.4 \\
\hline Hap & 139 & 107 & 1.30 & 114 & 139 \\
\hline Эмаль (Е) / Enamel & 114 & 66.9 & 1.70 & 96.2 & 114 \\
\hline Дентин (D) / Dentin & 36.2 & 17.5 & 2.07 & 28.9 & 36.2 \\
\hline \multicolumn{6}{|c|}{ Модуль сдвига / Shear modulus } \\
\hline Материал / Material & $G_{\max }, \mathrm{GPa}$ & $G_{\text {min }}, \mathrm{GPa}$ & $G_{\max } / G_{\min }$ & $G_{[01 \overline{1} 0]}, \mathrm{GPa}$ & $G_{[0001]}, \mathrm{GPa}$ \\
\hline Кость (B) / Bone & 10.2 & 6.21 & 1.64 & 8.16 & 6.21 \\
\hline Hap & 49.0 & 39.5 & 1.24 & 47.2 & 39.5 \\
\hline Эмаль (E) / Enamel & 44.2 & 22.8 & 1.94 & 36.2 & 22.8 \\
\hline Дентин (D) / Dentin & 14.0 & 5.70 & 2.46 & 10.2 & 5.70 \\
\hline
\end{tabular}
часто используемых для однофазных поликристаллов. Отметим, что приведенные рассчитанные значения достаточно хорошо согласуются с известными опытными данными.

Табл. 4. Экстремальные значения модуля Юнга и модуля сдвига и значения в частных направлениях, лежащих в плоскости (2$\overline{1} \overline{1} 0)$. Table 4. Extremal values of Young's modulus and shear modulus and values in the particular directions lying in the $(2 \overline{1} \overline{1} 0)$ plane.

Табл. 5. Значения усредненных упругих модулей.

Table 5. The values of the averaged elastic moduli.

\begin{tabular}{|c|c|c|c|c|c|c|c|c|}
\hline Материал / Material & $\left\langle K_{\mathrm{V}}>, \mathrm{GPa}\right.$ & $\left\langle G_{\mathrm{V}}>, \mathrm{GPa}\right.$ & $\left\langle K_{\mathrm{R}}>, \mathrm{GPa}\right.$ & $\left\langle G_{\mathrm{R}}>, \mathrm{GPa}\right.$ & $\left\langle K_{\mathrm{H}}>, \mathrm{GPa}\right.$ & $\left\langle G_{\mathrm{H}}\right\rangle, \mathrm{GPa}$ & $\langle E\rangle, \mathrm{GPa}$ & $\langle v>$ \\
\hline Кость (В) / Bone & 18.8 & 7.96 & 18.8 & 7.60 & 18.8 & 7.78 & 20.5 & 0.32 \\
\hline Нар & 83.4 & 44.9 & 81.8 & 44.3 & 82.6 & 44.6 & 113 & 0.27 \\
\hline Эмаль (Е) / Enamel & 62.2 & 33.2 & 62.2 & 30.5 & 62.2 & 31.9 & 81.7 & 0.28 \\
\hline Дентин (D) / Dentin & 20.1 & 9.59 & 20.0 & 8.21 & 20.1 & 8.90 & 23.3 & 0.31 \\
\hline
\end{tabular}




\section{6. Заключение}

Исследованы упругие свойства эмали и дентина, а также дано сравнение с упругими свойствами костной ткани и гидроксиапатита. Поведение коэффициентов Пуассона дентина и эмали оказалось подобным, а значения меняются в пределе от 0.13 и до 0.54 для дентина и от 0.16 и до 0.47 для эмали. Анализ модуля Юнга, модуля сдвига и параметров анизотропии для дентина, эмали, костной ткани и гидроксиапатита показал, что наибольшая анизотропия выявляется у дентина и эмали, меньшая у кости и наименьшая - у гидроксиапатита. Наибольшими значениями модуля Юнга и модуля сдвига обладают эмаль и гидроксиапатит. Полученные данные будут способствовать лучшему пониманию вопроса о взаимосвязи архитектоники зуба и его механических свойств, проблем прочности биокомпозитов, а также оптимизации решения задач биомеханики зубочелюстной системы с учетом упругой симметрии составляющих её твердых тканей.

Благодарности/Acknowledgements. Д.С.Л. выполнил работу в рамках Государственного задания AAAA-A17-117021310373-3./The work (D.S.L.) was financially supported by the Federal Agency for Scientific Organizations (Project AAAA-A17-117021310373-3).

\section{Литература/References}

1. J.L. Katz, K. Ukraincik. J. Biomech. 4, 221 (1971). DOI: 10.1016/0021-9290(71)90007-8

2. J.L. Katz. J. Biomech. 4, 455 (1971). DOI: 10.1016/0021-9290(71)90064-9

3. I.Yu. Lebedenko, S.D. Arutyunov, S.A. Muslov, A.S. Useynov. Cathedra. 32, 24 (2010). (in Russian) [И.Ю. Лебеденко, С.Д. Арутюнов, С.А. Муслов, А. С. Усеинов. Кафедра. 32, 24 (2010).]

4. I. Yu. Lebedenko, S.D. Arutyunov, S.A. Muslov, A.S. Useynov. Vestnik Rossiiskogo universiteta druzhby narodov. Seriya: Meditsina. 4, 637 (2009). (in Russian) [И.Ю. Лебеденко, С.Д. Арутюнов, С.А. Муслов, А. С. Усеинов. Вестник РУДН, серия Медицина. 4, 637 (2009).]

5. J. F. Nye. Physical Properties of Crystals. Clarendon Press, Oxford (1957) 329 p.

6. Yu.I. Sirotin, M.P. Shaskolskaya. Fundamentals of Crystal Physics. Mir, Moscow (1982) 640 p. (in Russian) [Ю.И. Сиротин, М.П. Шаскольская. Основы кристаллофизики. Москва, Наука (1979) 640 с.]

7. L. D. Landau, E. M. Lifshitz. Theory of Elasticity. Vol. 7. Course of Theoretical Physics. Butterworth-Heinemann
(1986) 195 p.

8. T.-C. Lim. Auxetic Materials and Structures. Springer Singapore (2015) 588 p. DOI: 10.1007/978-981-287-275-3

9. R.V. Goldstein, V.A. Gorodtsov, D.S. Lisovenko, M. A. Volkov. Phys. Mesomech. 17, 97 (2014). DOI: $10.1134 /$ S1029959914020027

10. H.M. A. Kolken, A.A. Zadpoor. RSC Adv. 7(9), 5111 (2017). DOI: 10.1039/C6RA27333E

11. R. V. Goldstein, D.S. Lisovenko, A.V. Chentsov, S. Yu. Lavrentyev. Letters on materials. 7(4), 355 (2017). DOI: 10.22226/2410-3535-2017-4-355-358

12. M. Bilski, K.W. Wojciechowski. Phys. Status Solidi B. 253(7), 1318 (2016). DOI: 10.1002/pssb.201600140

13. R.V. Goldstein, V.A. Gorodtsov, D.S. Lisovenko, M. A. Volkov. Letters on materials. 6(2), 93 (2016). DOI: $10.22226 / 2410-3535-2016-2-93-97$

14. S. S. Pertsov, G. M. Styureva, S. A. Muslov, A. A. Sinitsyn, A. A. Korneev, N.V. Zaitseva. Fundamentals of biomechanics for dentists. Moscow, MGMSU (2016) 109 p. (in Russian) [С. С. Перцов, Г. М. Стюрева, С. А. Муслов, А. А. Синицын, А. А. Корнеев, Н. В. Зайцева. Основы биомеханики для стоматологов. Москва, МГМСУ (2017) 109 c.]

15. S. Lees, F. Rollins. J. Biomech. 5, 557 (1972). DOI: 10.1016/0021-9290(72)90027-9

16. R.V. Goldstein, V.A. Gorodtsov, D.S. Lisovenko. Dokl. Phys. 56(12), 602 (2011). DOI: $10.1134 /$ S1028335811120019

17. R. V. Goldstein, V.A. Gorodtsov, M.A. Komarova, D.S. Lisovenko. Scripta Mater. 140, 55 (2017). DOI: 10.1016/j.scriptamat.2017.07.002

18. T.D. Shermergor. The theory of elasticity of microinhomogeneous media. Moscow, Nauka (1977) 400 р. [Т. Д. Шермергор. Теория упругости микронеоднородных сред. Москва, Наука (1977) 400 с.]

19. E. P. Bogdanov, I.A. Skoda. Relative parameters of elastic anisotropy for crystals with bcc and hcp lattice: Proceedings of "Progressive technologies in training and production". KTI VolgGTU (2006) P. 49 - 51. (in Russian) [Е.П. Богданов, И.А. Шкода. Относительные параметры упругой анизотропии для кристаллов с ОЦК и ГПУ решёткой: Материалы IV Всероссийской конференции "Прогрессивные технологии в обучении и производстве". КТИ ВолгГТУ (2006) С. 49 - 51.]

20. T.P. Chernyaeva, V.M. Gritsina, E.A. Mikhailov, A.V. Ostapov. Voprosy atomnoy nauki i tekhniki. Series: Phys. Rad. Povr. Rad. Material. 4-2, 206 (2009). (in Russian) [Т.П. Черняева, В.М.Грицина, Е.А. Михайлов, А.В. Остапов. Вопросы атомной науки и техники. Серия: Физ. Рад. Повр. Рад. Материал. 4-2, 206 (2009).] 